\title{
Bi-slant submanifolds of an $S$ manifold
}

\author{
Aykut Akgün \\ Technical Sciences Vocational High School, Adiyaman Universitesi, \\ Adiyaman, Turkey, and \\ Mehmet Gülbahar \\ Mathematics, Harran Universitesi, Sanliurfa, Turkey
}

Abstract

Purpose-Bi-slant submanifolds of $S$-manifolds are introduced, and some examples of these submanifolds are presented.

Design/methodology/approach - Some properties of $D_{i}$-geodesic and $D_{i}$-umbilical bi-slant submanifolds are examined.

Findings - The Riemannian curvature invariants of these submanifolds are computed, and some results are discussed with the help of these invariants.

Originality/value - The topic is original, and the manuscript has not been submitted to any other journal.

Keywords Submanifold, S-Manifold, Curvature

Paper type Research paper

\section{Introduction}

Slant submanifolds were firstly introduced by B. Y. Chen as a generalization of invariant and anti-invariant submanifolds of Kaehler manifolds and initial computations, results and examples of these kinds of submanifolds were presented in his book [1]. A submanifold $M$ of an almost Hermitian manifold involving an almost complex structure $J$ is called a slant submanifold if the angle between $J X_{p}$ and $X_{p}$ is independent of choosing of point $p \in M$ and every non-zero tangent vector $X_{p}$. Later, the concept of slant submanifolds has been drawing attentions of many geometers and these submanifolds have been studying in various space forms admitting complex, contact and product structures [2-6] etc.

From the definition of slant submanifolds, the concept of slanting can be carried to distributions in the tangent bundle on a Riemannian manifold. A smooth distribution $\mathfrak{D}$ is called as a slant distribution if the angle between $J \mathfrak{D}$ and $\mathfrak{D}$ is constant. By using slant distributions, bi-slant submanifolds of almost Hermitian manifolds were defined by A. Carriazo $[7,8]$.

A submanifold $M$ of an almost Hermitian manifold is called a bi-slant submanifold if there exist two orthogonal slant distributions, $\mathfrak{D}_{1}$ and $\mathfrak{D}_{2}$, on tangent bundle $T M$ of $M$ with slant angles $\theta_{1}$ and $\theta_{2}$, respectively, such that one writes

$$
T M=\mathfrak{D}_{1} \oplus \mathfrak{D}_{2} .
$$

Here, $\oplus$ denotes the orthogonal direct sum.

(C) Aykut Akgün and Mehmet Gülbahar. Published in Arab Journal of Mathematical Sciences. Published by Emerald Publishing Limited. This article is published under the Creative Commons Attribution (CC BY 4.0) licence. Anyone may reproduce, distribute, translate and create derivative works of this article (for both commercial and non-commercial purposes), subject to full attribution to the original publication and authors. The full terms of this licence may be seen at http://creativecommons.org/licences/by/4.0/ legalcode

The authors are thankful to the referees for their valuable comments towards the improvement of the paper.

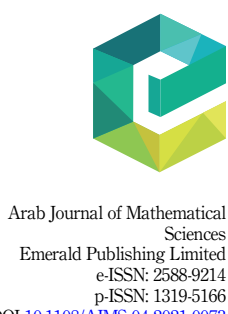

DOI 10.1108/ATMS-04: 1319-5166 
In the literature, there exist very interesting works on bi-slant submanifolds of various spaces [9-14]. An important aspect of slant submanifolds is that they can be considered as a generalization of semi-slant, hemi-slant and $C R$ submanifolds. In fact, a bi-slant submanifold becomes.

(1) a semi-slant submanifold if and only if $\theta_{1}=0$ (or $\theta_{2}=0$ ),

(2) a hemi-slant submanifold if and only if $\theta_{1}=\frac{\pi}{2}$ (or $\theta_{2}=\frac{\pi}{2}$,

(3) a CR-submanifold if and only if $\theta_{1}=0$ and $\theta_{2}=\frac{\pi}{2}$.

One of the important points about bi-slant submanifolds is deal to the relations between bi-slant submanifolds and slant submanifolds. Although slant submanifolds may seem like a special case of bi-slant submanifolds at first glance, this information is not correct in general. It should be noted that a bi-slant submanifold may not be a slant submanifold even if $\theta_{1}=\theta_{2}$. Another point to note that one cannot know the angle between $J \mathfrak{D}_{1}$ and $\mathfrak{D}_{2}$ for any bi-slant submanifolds. Furthermore, any invariant submanifold does not have to be a bi-slant submanifold even if $\theta_{1}=\theta_{2}=0$.

On the other hand, one of the most fundamental problems in submanifold theory is to establish main relations between the extrinsic and intrinsic curvature invariants for submanifolds. In this respect, B. Y. Chen [15] established an inequality involving Ricci curvature and the squared mean curvature known as the Chen-Ricci inequality in the literature. Later, this inequality has been investigated for submanifolds of ambient spaces $\mathrm{cf}$ [16-20].

Considering the facts mentioned above, we investigate bi-slant submanifolds of metric $f$ manifolds and $S$-manifolds in this paper. We give some examples and investigate to totally geodesic and totally umbilical submanifolds of bi-slant submanifolds. Furthermore, we compute the curvature tensors and examine the Chen-Ricci inequality and its results on these submanifolds.

\section{Preliminaries}

Let $(\widetilde{M}, \widetilde{g})$ be an $m$-dimensional Riemannian manifold with a Riemannian metric $\widetilde{g}$ and $\left\{e_{1}\right.$, $\left.\ldots, e_{m}\right\}$ be an orthonormal basis for $T_{p} \widetilde{M}$ at a point $p \in \widetilde{M}$. The Ricci curvature $\widetilde{\text { Ric }}$ is defined by

$$
\widetilde{\operatorname{Ric}}(X, Y)=\sum_{j=1}^{m} \widetilde{g}\left(\widetilde{R}\left(e_{j}, X\right) Y, e_{j}\right)
$$

for any $X, Y \in T \widetilde{M}$. For a fixed $i \in\{1, \ldots, m\}$, we have

$$
\widetilde{\operatorname{Ric}}\left(e_{i}, e_{i}\right) \equiv \widetilde{\operatorname{Ric}}\left(e_{i}\right)=\sum_{j \neq i}^{m} \widetilde{K}\left(e_{i}, e_{j}\right) \text {. }
$$

Here, $\widetilde{K}\left(e_{i}, e_{j}\right)$ denotes the sectional curvature of the plane section spanned by $e_{i}$ and $e_{j}$ in $T \widetilde{M}$.

Let $\Pi_{k}$ be a $k$-plane subsection of $T_{p} \widetilde{M}$ and $X$ be a unit vector in $\Pi_{k}$. We choose an orthonormal basis $\left\{e_{1}, \ldots, e_{k}\right\}$ of $\Pi_{k}$ such that $e_{1}=X$. Then, the Ricci curvature $\widetilde{\operatorname{Ric}}_{\Pi_{k}}$ of $\Pi_{k}$ at $X$ is defined by

$$
\widetilde{\operatorname{Ric}}_{\Pi_{k}}(X)=\widetilde{K}_{12}+\widetilde{K}_{13}+\cdots+\widetilde{K}\left(e_{1}, e_{k}\right)
$$


Here, $\widetilde{\operatorname{Ric}}_{\Pi_{k}}(X)$ is called as $k$-Ricci curvature of $X[15]$. Thus for each fixed $e_{i}, i \in\{1, \ldots, k\}$ we get

$$
\widetilde{\operatorname{Ric}}_{\Pi_{k}}\left(e_{i}\right)=\sum_{j \neq i}^{k} \widetilde{K}\left(e_{i}, e_{j}\right)
$$

Let $(M, g)$ be an $n$-dimensional submanifold of an $m$-dimensional Riemannian manifold $(\widetilde{M}, \widetilde{g})$ with the induced metric $g$ from $\widetilde{g}$. The Gauss and Weingarten formulas are given by

$$
\widetilde{\nabla}_{X} Y=\nabla_{X} Y+h(X, Y) \quad \text { and } \quad \widetilde{\nabla}_{X} N=-A_{N} X+\nabla_{X}^{\perp} N
$$

for all $X, Y \in T M$ and $N \in T^{\perp} M$. Here, $\widetilde{\nabla}, \nabla$ and $\nabla^{\perp}$ are, respectively, the Riemannian, induced Riemannian and induced normal connections in $\widetilde{M}, M$ and the normal bundle $T^{\perp} M$ of $M$ and $h$ is the second fundamental form related to the shape operator $A$ by

$$
\widetilde{g}(h(X, Y), N)=g\left(A_{N} X, Y\right) .
$$

The mean curvature vector $H$ is given by $H=\frac{1}{n} \operatorname{trace}(h)$. The submanifold $M$ is called totally geodesic in $\widetilde{M}$ if $h=0$ and minimal if $H=0$. If $h(X, Y)=g(X, Y) H$ for all $X, Y \in T M$, then $M$ is called totally umbilical [21].

Let $R$ and $\widetilde{R}$ denote the Riemannian curvature tensor fields of $M$ and $\widetilde{M}$ respectively. The well-known equation of Gauss is given by

$$
\begin{aligned}
g(R(X, Y) Z, W)= & \widetilde{g}(\widetilde{R}(X, Y) Z, W)+\widetilde{g}(h(X, W), h(Y, Z)) \\
& -\widetilde{g}(h(X, Z), h(Y, W))
\end{aligned}
$$

for all $X, Y, Z, W \in T M$.

Now, we shall recall the Chen-Ricci inequality in the following:

Theorem 2.1. [[22], Theorem 6.1] Let $M$ be an n-dimensional submanifold of a Riemannian manifold. Then, the following statements are true.

(1) For any unit vector field $X \in T M$, it follows that

$$
\operatorname{Ric}(X) \leq \frac{1}{4} n^{2}\|H\|^{2}+\widetilde{\operatorname{Ric}}_{\left(T_{p} M\right)}(X),
$$

where $\widetilde{\operatorname{Ric}}_{\left(T_{p} M\right)}(X)$ is the n-Ricci curvature of the tangent space $T_{p} M$ of X at $p \in M$ with respect to the ambient manifold $\widetilde{M}$.

(2) The equality case of (2.7 is satisfied by for a unit vector field $X \in T_{p} M$ if and only if

$$
\left\{\begin{array}{l}
h(X, Y)=0, \\
2 h(X, X)=n H(p) .
\end{array} \quad \text { for all } Y \in T_{p} M \text { orthogonal to } X,\right.
$$

(3) The equality case of (2.7) holds for all unit tangent vector $X \in T_{p} M$ if and only if either $p$ is a totally geodesic point or $n=2$ and $p$ is a totally umbilical point.

\section{Metric $f$-manifolds and their submanifolds}

A Riemannian manifold $(\widetilde{M}, \widetilde{g})$ is called as a metric $f$-manifold if there exists an $f$ structure $\left(f, \xi_{1}, \ldots, \xi_{s}, \eta_{1}, \ldots, \eta_{s}\right)$ consisting of a tensor field $f$ of type $(1,1)$, structure vector fields $\xi_{1}$, $\ldots, \xi_{s}$ and 1 -forms $\eta_{1}, \ldots, \eta_{s}$ such that this structure satisfies [23]. 


$$
f^{2}=-I+\sum_{\alpha=1}^{s} \eta_{\alpha} \otimes \xi_{\alpha}, \quad \eta_{\alpha}\left(\xi_{\alpha}\right)=1, \quad f \xi=0, \quad \eta_{\alpha} \circ f=0
$$

for any $\alpha \in\{1, \ldots, s\}$ and

$$
\widetilde{g}(X, Y)=\widetilde{g}(f X, f Y)+\sum_{\alpha=1}^{s} \eta_{\alpha}(X) \eta_{\alpha}(Y) .
$$

for any $X, Y \in T \widetilde{M}$. We note that the first and one of the remaining relations of (3.1) imply the other two relations.

The condition for an $f$ structure being normal is equivalent to vanishing of the torsion tensor such that we have

$$
[f, f]+2 \sum_{\alpha=1}^{s} d \eta_{\alpha} \otimes \xi_{\alpha}
$$

where $[f, f]$ is the Nijenhuis tensor of $f$, given by

$$
[f, f](X, Y)=[f X, f Y]-f[f X, Y]-f[X, f Y]+f^{2}[X, Y] .
$$

Let $F$ denotes the fundamental 2 -form which is defined by

$$
F(X, Y)=\widetilde{g}(X, f Y)
$$

for any $X, Y \in T \widetilde{M}$. An $f$ metric structure $\left(f, \xi_{\alpha}, \varphi \alpha, \widetilde{g}\right)$ becomes an $S$ manifold if $F=d \eta$; and the $f$ structure is normal. For an $S$ manifold, it is known that the following relation holds for any $X \in T \widetilde{M}$ and $\alpha \in\{1, \ldots, s\}$

$$
\widetilde{\nabla}_{X} \xi_{\alpha}=-f X .
$$

Now, suppose that $\mathfrak{\Omega}$ to be a distribution spanned by the structure vector fields $\xi_{1}, \ldots, \xi_{s}$ of an $S$ manifold and $D$ be its complementary orthogonal distribution. In this case, we can write

$$
T M=\mathfrak{D} \oplus \mathfrak{R} .
$$

Hence, it is clear that if $X \in \mathcal{Q}$ then $\eta_{\alpha}(X)=0$ for any $\alpha=\{1, \ldots, s\}$ and if $X \in \mathfrak{D}$ then $f X=0$.

Let $\Pi$ be a plane section spanned by $X$ and $f X$ for any $X \in \mathfrak{D}$. Then this plane is called an $f$ section of $\mathfrak{D}$ and the sectional curvature of such a plane is called as $f$ sectional curvature. An $S$ manifold whose all of $f$ sectional curvatures are a constant value $c$ is said to be an $S$ space form. The Riemann curvature tensor of an $S$ space form of constant $f$ sectional curvature $c$ is given by

$$
\begin{aligned}
\widetilde{g}(\widetilde{R}(X, Y) Z, W))= & \frac{c}{4}\{g(f X, f W) g(f Y, f Z)-g(f X, f Z) g(f Y, f W) \\
& +g(X, f W) g(Y, f Z)-g(X, f Z) g(Y, f W) \\
& -2 g(X, f Y) g(Z, f W)\}
\end{aligned}
$$

for all $X, Y, Z, W \in T \widetilde{M}[24]$.

Next, let $M$ be a submanifold of a metric $f$ manifold. For any $X \in T M$, we put

$$
f X=T X+N X,
$$

where $T X$ is the tangential part and $N X$ is the normal part of $f X$ respectively. In a similar way, for any vector field normal to $M$, we put 


$$
f V=t V+n V
$$

where $t V$ and $n V$ are the tangential and normal parts of $f V$ respectively.

From (3.2), (3.3) and (3.7), it is easy to see that

$$
g(T X, Y)=-g(X, T Y)
$$

for any $X, Y \in T M$.

A submanifold $M$ is said to be an invariant submanifold (resp. anti-invariant) if $T=0$ (resp. $N=0$ ) [25]. For each non-zero vector field $X$ in $T M$, if the angle between $f X$ and $T X$ is independent of the choice of $p \in M$ and $X$ then $M$ is called a slant submanifold. Note that $M$ is a slant submanifold if and only if there exists a constant $\lambda \in[0,1]$ satisfying

$$
T^{2}=-\lambda I+\lambda \sum_{\alpha=1}^{s} \eta_{\alpha} \otimes \xi_{\alpha}
$$

where $I$ denotes the identity map [26].

\section{Bi-slant submanifolds}

Let $M$ be a submanifold of an $S$ manifold and $\xi_{1}, \ldots, \xi_{s}$ to be tangent to $M$. A smooth distribution (D) on $M$ is called a slant distribution if the angle between $X$ and $f X$ is constant for each non-zero vector $X$ in $\mathfrak{D}_{p}$ and for each $p \in M$.

Next, we suppose that $P$ is the projection to $T M$ onto $\mathfrak{D}$. In a similar way to (3.10) it can be also proved that $\mathfrak{D}$ is a slant distribution if and only if there exists a constant $\lambda \in[0,1]$ satisfying

$$
(P T)^{2}=-\lambda I+\lambda \sum_{\alpha=1}^{s} \eta_{\alpha} \otimes \xi_{\alpha}
$$

Definition 4.1. For a submanifold $M$ of an $S$ manifold $\widetilde{M}$ we say that $M$ is a bi-slant submanifold of $\widetilde{M}$ if there exist there orthogonal distributions $\mathfrak{D}_{1}, \mathfrak{D}_{2}$ and $\mathbb{2}$ such that

(1) $T M=\mathfrak{D}_{1} \oplus \mathfrak{D}_{2} \oplus \mathfrak{\Omega}$

(2) For any $i \in\{1,2\}$, D $i$ is a slant distribution with slant angle $\theta_{i}$.

Now we shall give some examples of bi-slant submanifolds of $S$ manifolds.

Example 4.2. Let $E^{8+s}$ denotes the Euclidean $(8+s)$ space with Cartesian coordinates $\left(x^{1}, x^{2}, x^{3}, x^{4}, y^{1}, y^{2}, y^{3}, y^{4}, z^{1}, \ldots, z^{s}\right)$. An S structure on $E^{8+s}$ is usually given by the following equations:

$$
\begin{aligned}
& \eta_{\alpha}=\frac{1}{2}\left(d z^{\alpha} \sum_{i=1}^{4} y^{i} d x^{i}\right), \quad \xi_{\alpha}=2 \frac{\partial}{\partial z^{\alpha}} \\
& g=\sum_{\alpha=1}^{s} \eta_{\alpha} \otimes \eta_{\alpha}+\frac{1}{8} \sum_{i=1}^{4} d x^{i} \otimes+d x^{i}+d y^{i} \otimes+d y^{i} \\
& f\left(\sum_{i=1}^{4}\left(X_{i} \frac{\partial}{\partial x^{i}}+Y_{i} \frac{\partial}{\partial y^{i}}\right)+\sum_{\alpha=1}^{s} Z_{\alpha} \frac{\partial}{\partial z^{\alpha}}\right)=\sum_{i=1}^{4}\left(Y_{i} \frac{\partial}{\partial x^{i}}-X_{i} \frac{\partial}{\partial y^{i}}\right)+\sum_{\alpha=1}^{s} Y_{i} y^{i} \frac{\partial}{\partial z^{\alpha}}
\end{aligned}
$$




$$
x\left(u, v, w, m, t_{1}, t_{2}, \ldots, t_{s}\right)=\left(u \cos \theta_{1}, u \sin \theta_{1}, w \cos \theta_{2}, w \sin \theta_{2}, v, 0, m, 0, t_{1}, t_{2}, \ldots, t_{s}\right)
$$

for any $\theta \in\left[0, \frac{\Pi}{2}\right]$. Then we obtain a basis of TM as follows:

$$
\begin{aligned}
e_{1} & =\frac{\partial}{\partial u}+\sum_{\alpha=1}^{s} \cos \theta_{1} v \frac{\partial}{\partial t_{\alpha}} \\
& =\cos \theta_{1}\left(\frac{\partial}{\partial x_{1}}+\sum_{\alpha=1}^{s} y^{1} \frac{\partial}{\partial z_{\alpha}}\right)+\sin \theta_{1}\left(\frac{\partial}{\partial x_{1}}+\sum_{\alpha=1}^{s} y^{2} \frac{\partial}{\partial z_{\alpha}}\right), \\
e_{2} & =\frac{\partial}{\partial v}=\frac{\partial}{\partial y_{1}}, \\
e_{3} & =\frac{\partial}{\partial w}+\sum_{\alpha=1}^{s} \cos \theta_{2} m \frac{\partial}{\partial t_{\alpha}} \\
& =\cos \theta_{2}\left(\frac{\partial}{\partial x_{3}}+\sum_{\alpha=1}^{s} y^{3} \frac{\partial}{\partial z_{\alpha}}\right)+\sin \theta_{2}\left(\frac{\partial}{\partial x_{3}}+\sum_{\alpha=1}^{s} y^{4} \frac{\partial}{\partial z_{\alpha}}\right), \\
e_{4} & =\frac{\partial}{\partial m}, \\
e_{4+\alpha} & =\frac{\partial}{\partial t_{\alpha}}=2 \frac{\partial}{\partial z_{\alpha}}=\xi_{\alpha}, \text { for any } \alpha=1,2, \ldots, s .
\end{aligned}
$$

Suppose that $\mathfrak{D}_{1}=\operatorname{Span}\left\{e_{1}, e_{2}\right\}, \mathfrak{D}_{2}=\operatorname{Span}\left\{e_{3}, e_{4}\right\}$ and $\mathfrak{Q}=\operatorname{Span}\left\{e_{4+1}, \ldots, e_{4+s}\right\}$. Then we see that $\mathrm{M}$ is a bi-slant submanifold with angle $\left(\theta_{1}, \theta_{2}\right)$. Moreover, the vector fields

$$
\begin{aligned}
& e_{5+s}=-\sin \theta_{1}\left(\frac{\partial}{\partial x_{1}}+\sum_{\alpha=1}^{s} y^{1} \frac{\partial}{\partial z_{\alpha}}\right)+\cos \theta_{1}\left(\frac{\partial}{\partial x_{1}}+\sum_{\alpha=1}^{s} y^{2} \frac{\partial}{\partial z_{\alpha}}\right), \\
& e_{6+s}=\frac{\partial}{\partial y^{2}} \\
& e_{7+s}=-\sin \theta_{2}\left(\frac{\partial}{\partial x_{3}}+\sum_{\alpha=1}^{s} y^{3} \frac{\partial}{\partial z_{\alpha}}\right)+\cos \theta_{2}\left(\frac{\partial}{\partial x_{4}}+\sum_{\alpha=1}^{s} y^{4} \frac{\partial}{\partial z_{\alpha}}\right), \\
& e_{8+s}=\frac{\partial}{\partial y^{4}}
\end{aligned}
$$

form a basis of the normal space $T^{\perp} M$. Also, it can be shown that both bases are orthonormal. Example 4.3. Consider the Euclidean 8-space $E^{8+s}$ with the usual $S$ structure given in Example 4.2. For any constant $k$,

$$
\begin{aligned}
x\left(u, v, w, m, t_{1}, \ldots, t_{s}\right)= & 2\left(e^{k u} \cos u \cos v, e^{k u} \sin u \cos v, e^{k u} \cos u \sin v,\right. \\
& e^{k u} \sin u \sin v, e^{k u} \cos w \cos m, e^{k u} \sin w \cos m, \\
& \left.e^{k u} \cos w \sin m, e^{k u} \sin w \sin m, t_{1}, \ldots, t_{s}\right)
\end{aligned}
$$


defines a $(4+s)$-dimensional bi-slant submanifold $M$ with the bi-slant angles $\left(\theta_{1}, \theta_{2}\right)$ such that

$$
\theta_{1}=\arccos \frac{k}{\sqrt{2 k^{2}+1}}
$$

and

$$
\theta_{2}=\arccos \frac{-k}{\sqrt{k^{2}+1}}
$$

Further examples could be given.

Let $P_{i}: T M \rightarrow D_{i}$ be projections. Then we can write any vector field $X$ in $T M$ by these projections

$$
X=P_{1} X+P_{2} X+\sum_{\alpha=1}^{3} \eta_{\alpha}(X) \xi_{\alpha}
$$

and

$$
\begin{aligned}
\phi X & =T X+N X \\
& =P_{1} T X+P_{2} T X+\sum_{\alpha=1}^{3} \eta_{\alpha}(X) \xi_{\alpha}+N X
\end{aligned}
$$

Following the proof way of equation (3.8) in Ref. [10] and using the above facts, we get the following theorem:

Theorem 4.4. Let $M$ be $a(2 n+2 m+s)$-dimensional bi-slant submanifold of $\tilde{M}$. Then there exists an orthonormal frame field $\left\{e_{1}, \ldots, e_{2 n}, e_{2 n+1}, \ldots, e_{2 n+2 m}, \xi_{1}, \ldots, \xi_{s}\right\}$ on TM where $\mathfrak{D}_{1}=\operatorname{Span}\left\{e_{1}, \ldots, e_{2 n}\right\}, \mathfrak{D}_{2}=\operatorname{Span}\left\{e_{2 n+1}, \ldots, e_{2 n+2 m}\right\}$,

$\mathfrak{Q}=\operatorname{Span}\left\{\xi_{1}, \ldots, \xi_{s}\right\}$ such that the following conditions hold:

$$
\begin{aligned}
T e_{1} & =\cos \theta_{1} e_{2}+P_{2} T e_{1} \\
T e_{2} & =-\cos \theta_{1} e_{1}+P_{2} T e_{2}, \\
& \vdots \\
T e_{2 n} & =-\cos \theta_{1} e_{2 n-1}+P_{2} T e_{2 n}
\end{aligned}
$$

and

$$
\begin{aligned}
T e_{2 n+1} & =\cos \theta_{2} e_{2 n+2}+P_{1} T e_{2 n+1}, \\
T e_{2 n+2} & =-\cos \theta_{2} e_{2 n+1}+P_{1} T e_{2 n+2}, \\
& \vdots \\
T e_{2 n+2 m} & =-\cos \theta_{2} e_{2 n+2 m-1}+P_{1} T e_{2 n+2 m} .
\end{aligned}
$$

Considering (4.1) and Theorem 4.4, we obtain the following lemmas immediately: 
Lemma 4.5. Let $M$ be a bi-slant submanifold of $\widetilde{M}$. we can write

$$
g\left(T P_{i} X, T P_{i} Y\right)=\cos ^{2} \theta_{i}\left(g\left(P_{i} X, P_{i} Y\right)-\sum_{\alpha=1}^{3} \eta_{\alpha}\left(T P_{i} X\right) \eta_{\alpha}\left(T P_{i} Y\right)\right)
$$

for any $X, Y \in T M$.

Lemma 4.6. Any totally geodesic or totally umbilical bi-slant submanifold of an S manifold is an invariant submanifold.

Proof. From (3.4) and (3.7), we have for any $X$ in $T M$ and $\alpha \in\{1, \ldots, s\}$

$$
\widetilde{\nabla}_{X} \xi_{\alpha}=-T X+N X
$$

which shows that

$$
h\left(X, \xi_{\alpha}\right)=-N X
$$

Hence, if $M$ is totally geodesic or then it is clear that $N=0$ which shows that the submanifold is invariant.

From Theorem 4.6, we see that the study of totally geodesic or totally umbilical bi-slant submanifolds of an $S$ manifold reduces to the study of invariant submanifolds. Therefore, we shall investigate to the concepts of $D_{i}$ geodesic or $D_{i}$ totally umbilical bi-slant submanifolds of an $S$ manifold throughout this study.

Now, let us consider any two vector fields $X$ and $Y$ in $T M$ such that we write

$$
X=P_{1} X+P_{2} X+\sum_{\alpha=1}^{s} \eta(X) \xi_{\alpha} \text { and } Y=P_{1} Y+P_{2} Y+\sum_{\alpha=1}^{s} \eta(Y) \xi_{\alpha} .
$$

If $M$ is $D_{i}$ geodesic, then we have $h\left(P_{i} X, P_{i} Y\right)=0$. Using this fact we see that the submanifold is totally $D_{i}$ geodesic if and only if

$$
\begin{aligned}
h(X, Y)= & h\left(P_{i} X, P_{j} Y\right)+h\left(P_{j} X, P_{i} Y\right)+h\left(P_{j} X, P_{j} Y\right) \\
& +\sum_{\alpha=1}^{s} \eta_{\alpha}(X) h\left(Y, \xi_{\alpha}\right)+\eta_{\alpha}(Y) h\left(X, \xi_{\alpha}\right) .
\end{aligned}
$$

If $M$ is totally $D_{i}$ umbilical if and only if

$$
\begin{aligned}
h(X, Y)= & h\left(P_{i} X, P_{j} Y\right)+h\left(P_{j} X, P_{i} Y\right)+h\left(P_{j} X, P_{j} Y\right) \\
& +\sum_{\alpha=1}^{s} \eta_{\alpha}(X) h\left(Y, \xi_{\alpha}\right)+\eta_{\alpha}(Y) h\left(X, \xi_{\alpha}\right)+g(f X, f Y)
\end{aligned}
$$

for $i \neq j \in\{1,2\}$.

Theorem 4.7. Let $M$ be $(2 n+2 m+s)$-dimensional $\left(\theta_{1}, \theta_{2}\right)$ bi-slant submanifold of an $S$ manifold. If $M$ is $\mathfrak{D}_{i}$-geodesic then we have the following relations:

(1) For any $X_{i} \in \mathfrak{D}_{i}$, we have $N X_{i}=0$.

(2) For any $i \neq j \in\{1,2\}$, we have

$$
P_{i} T P_{j} T=\sin ^{2} \theta_{i} I .
$$


Proof. From (3.4) and (3.7), the proof of the statement (1) is straightforward.

Now we prove the statement (2), Since $M$ is $\mathfrak{D}_{i}$-geodesic, we put

$$
\begin{aligned}
f P_{i} X & =T P_{i} X \\
& =P_{i} T P_{i} X+P_{j} T P_{i} X
\end{aligned}
$$

for any $X \in T M$. Using Lemma 4.5, we get

$$
\widetilde{g}\left(f P_{i} X, f P_{i} X\right)=\cos ^{2} \theta_{i} g\left(P_{i} X, P_{i} X\right)+g\left(P_{j} T P_{i} X, P_{j} T P_{i} X\right),
$$

which implies that

$$
g\left(P_{j} T P_{i} X, P_{j} T P_{i} X\right)=\sin ^{2} \theta_{i} g\left(P_{i} X, P_{i} X\right) .
$$

Hence we obtain

$$
g\left(P_{i} T P_{j} T P_{i} X, P_{i} X\right)=\sin ^{2} \theta_{i} g\left(P_{i} X, P_{i} X\right),
$$

which shows the proof of statement (2).

Theorem 4.8. Let $M$ be a $\left(\theta_{1}, \theta_{2}\right)$ bi-slant submanifold of $\widetilde{M}$. For any $\theta \in\left[0, \frac{\pi}{2}\right]$, $M$ is $\theta$-slant if and only if the following relations hold:

$$
\begin{gathered}
P_{2} T P_{1} T P_{1}+P_{2} T P_{2} T P_{1}=0, \\
P_{1} T P_{1} T P_{2}+P_{1} T P_{2} T P_{2}=0, \\
P_{1} T P_{2} T P_{1}=\left(\cos ^{2} \theta_{1}-\cos ^{2} \theta\right) P_{1}, \\
P_{2} T P_{1} T P_{2}=\left(\cos ^{2} \theta_{2}-\cos ^{2} \theta\right) P_{2} .
\end{gathered}
$$

Proof. Suppose that $M$ is $\theta$-slant and $\mathfrak{D}=\mathfrak{D}_{1} \oplus \mathfrak{D}_{2}$. Then we write

$$
T(T X)=T\left(P_{1} T X+P_{2} T X\right) .
$$

for any $X \in \mathfrak{D}$. Using (3.10), (4.1) and (4.14), we have

$$
\begin{aligned}
T(T X)= & -\cos ^{2} \theta_{1} P_{1} X+P_{1} T P_{1} T P_{2} X+P_{2} T P_{1} T P_{1} X \\
& +P_{2} T P_{1} T P_{2} X+P_{1} T P_{2} T P_{1} X+P_{1} T P_{2} T P_{2} X \\
& +P_{2} T P_{2} T P_{1} X-\cos ^{2} \theta_{2} P_{2} X
\end{aligned}
$$

Considering tangential parts of $\mathfrak{D}_{1}$ and $\mathfrak{D}_{2}$ in (4.15), the proof is straightforward. The converse can be obtained directly.

Theorem 4.9. Let $M$ be a $\mathfrak{D}_{i}$-geodesic $\left(\theta_{1}, \theta_{2}\right)$ bi-slant submanifold. If $M$ is $\theta$-slant for any $\theta \in\left[0, \frac{\pi}{2}\right]$, then $\theta_{1}=\theta_{2}$ and there exists the following equation for $i \in\{1,2\}$.

$$
\cos ^{2} \theta=\cos 2 \theta_{i}
$$


Proof. From (4.7), (4.12) and (4.13) we get

$$
\sin ^{2} \theta_{i}=\cos ^{2} \theta_{i}-\cos ^{2} \theta
$$

which implies (4.16). Since equation (4.16) satisfies for all $i \in\{1,2\}$, we obtain that $\theta_{1}=\theta_{2}$.

Remark 4.10. We note that if a $\left(\theta_{1}, \theta_{2}\right)$ bi-slant submanifold is $\theta$-slant, then the angle $\theta$ does not have to be equal to $\theta_{1}$ and $\theta_{2}$. For this situation, we refer to Example 4.3 of $A$. Carriazzo [7] and Example 3.3 of [10].

As a result of Theorem 4.9, we get the following:

Corollary 4.11. Let $M$ be a $\mathfrak{D}_{i}$-geodesic $\left(\theta_{1}, \theta_{2}\right)$ bi-slant submanifold. If $M$ is $\theta$-slant with $\theta=\theta_{1}=\theta_{2}$ then $M$ is an invariant submanifold.

Theorem 4.12. Let $M$ be a $\left(\theta_{1}, \theta_{2}\right)$ bi-slant submanifold. If $M$ is both $\mathfrak{D}_{1}$ and $\mathfrak{D}_{2}$ geodesic submanifold then $\theta_{1}+\theta_{2}=\frac{\pi}{2}$

Proof. Suppose $X$ is a unit vector field on $\mathfrak{D}$ such that $\mathfrak{D}=\mathfrak{D}_{1} \oplus \mathfrak{D}_{2}$. Considering the statement of (1) of Theorem 4.7 we put

$$
f X=T P_{1} X+P_{2} T X .
$$

Since $M$ is $\left(\theta_{1}, \theta_{2}\right)$ bi-slant, we write from Theorem 4.4 that

$$
f X=\cos \theta_{1} P_{1} X+\cos \theta_{2} P_{2} X .
$$

which implies that $\cos \theta_{2}=\sin \theta_{1}$. Therefore, we obtain $\theta_{1}+\theta_{2}=\frac{\pi}{2}$ which is the claim of theorem.

With similar arguments, we have the following theorem.

Theorem 4.13. Let $M$ be a $\left(\theta_{1}, \theta_{2}\right)$ bi-slant submanifold of an $S$ manifold. Then we have the following statements:

(1) If $M$ is both totally umbilical then it is an invariant submanifold.

(2) If $M$ is $\mathfrak{D}_{1}$ and $\mathfrak{D}_{2}$ totally umbilical, then $\theta_{1}+\theta_{2}=\frac{\pi}{2}$

As a result od Theorem 4.12 and Theorem 4.13, we obtain the following:

Corollary 4.14. There do not exist semi-slant and hemi-slant submanifolds of an Smanifold which is $\mathfrak{D}_{1}$ and $\mathfrak{D}_{2}$ geodesic or $\mathfrak{D}_{1}$ and $\mathfrak{D}_{2}$-totally umbilical.

\section{Ricci curvatures of bi-slant submanifolds}

In this section, we investigate the Chen-Ricci inequality and its results for bi-slant submanifolds of an $S$ space form.

We need the following lemma for later uses:

Lemma 5.1. Let Mbe a $(2 n+2 m+s)$-dimensional $\left(\theta_{1}, \theta_{2}\right)$ bi-slant submanifold of an $S$-space form and $\left\{e_{1}, \ldots, e_{2 n}, e_{2 n+1}, \ldots, e_{2 n+2 m}, \xi_{1}, \ldots, \xi_{s}\right\}$ be an orthonormal basis of TM such that $\mathfrak{D}_{1}=\operatorname{Span}\left\{e_{1}, \ldots, e_{2 n}\right\}$ and $\mathfrak{D}_{2}=\operatorname{Span}\left\{e_{2 n+1}, \ldots, e_{2 n+2 m}\right\}$. Then we have the following equalities:

(1) For any $k \neq \ell \in\{1, \ldots, 2 n+2 m\}, a \neq b \in\{1, \ldots, s\}$ and plane sections $\Pi_{k \ell}=\left\{e_{s}, e_{k}\right\}$, $\Pi_{k a}=\operatorname{Span}\left\{e_{k}, \xi_{a}\right\}$ and $\Pi_{a b}=\operatorname{Span}\left\{\xi_{a}, \xi_{b}\right\}$, we have

$$
\widetilde{K}\left(\Pi_{k \ell}\right)=\frac{c}{4}\left\{1+3 g\left(f e_{k}, e_{\ell}\right)^{2}\right\}
$$


and

$$
\widetilde{K}\left(\Pi_{k a}\right)=\widetilde{K}\left(\Pi_{a b}\right)=0 .
$$

(2) For any $i \in\{1, \ldots, 2 n\}$ and $j \in\{2 n+1, \ldots, 2 n+2 m\}$, we have

$$
\widetilde{\operatorname{Ric}}_{T_{p} M}\left(e_{i}\right)=\frac{c}{4}\left\{(2 n+2 m-1)+3 \cos ^{2} \theta_{1}+3\left\|P_{2} T e_{i}\right\|^{2}\right\}
$$

and

$$
\widetilde{\operatorname{Ric}}_{T_{p} M}\left(e_{j}\right)=\frac{c}{4}\left\{(2 n+2 m-1)+3 \cos ^{2} \theta_{2}+3\left\|P_{1} T e_{j}\right\|^{2}\right\} .
$$

Proof. Putting $X=W=e_{k}$ and $Y=Z=e_{\ell}$ in (3.6) we have

$$
\begin{aligned}
\left.\widetilde{g}\left(\widetilde{R}\left(e_{k}, e_{\ell}\right) e_{\ell}, e_{k}\right)\right)= & \frac{c}{4}\left\{g\left(f e_{k}, f e_{k}\right) g\left(f e_{\ell}, f e_{\ell}\right)-g\left(f e_{k}, f e_{\ell}\right) g\left(f e_{\ell}, f e_{k}\right)\right. \\
& +g\left(e_{k}, f e_{k}\right) g\left(e_{\ell}, f e_{\ell}\right)-g\left(e_{k}, f e_{\ell}\right) g\left(e_{\ell}, f e_{k}\right) \\
& \left.-2 g\left(e_{k}, f e_{\ell}\right) g\left(e_{\ell}, f e_{k}\right)\right\}
\end{aligned}
$$

From (3.9) and (5.5) we get (5.1). In a similar way, it can be obtained (5.2) which completes the proof of $(a)$. Using (2.2) and (5.1), we obtain (5.3) and (5.4) thus the proof of $(b)$ is straightforward.

Theorem 5.2. Let $M$ be a $(2 n+2 m+s)$-dimensional bi-slant submanifold of an S-space form. Then, the following statements are true.

(1) For any unit vector field $X \in \mathfrak{D}_{1}$, it follows that

$$
\operatorname{Ric}(X) \leq \frac{1}{4} n^{2}\|H\|^{2}+\frac{c}{4}\left\{(2 n+2 m-1)+3 \cos ^{2} \theta_{1}+\left\|P_{2} T X\right\|^{2}\right\} .
$$

The equality case of (5.6) holds for all unit tangent vector $X \in \mathfrak{D}_{1}$ if and only if $M$ is $\mathfrak{D}_{1}$ totally geodesic.

(2) For any unit vector field $X \in \mathfrak{D}_{2}$, it follows that

$$
\operatorname{Ric}(X) \leq \frac{1}{4} n^{2}\|H\|^{2}+\frac{c}{4}\left\{(2 n+2 m-1)+3 \cos ^{2} \theta_{2}+\left\|P_{1} T X\right\|^{2}\right\} .
$$

The equality case of (5.7) holds for all unit tangent vector $X \in \mathfrak{D}_{2}$ if and only if $M$ is $\mathfrak{D}_{2}$ totally geodesic.

(3) The equality cases of both (5.6) and (5.7) satisfy then $\theta_{1}+\theta_{2}=0$.

Proof. Putting (5.3) and (5.4) in (2.7), we obtain (5.6) and (5.7) inequalities respectively. Considering the (3) statements of Theorem 2.1 we get $M$ is $\mathfrak{D}_{1}$ and $\mathfrak{D}_{2}$ geodesic. Using this fact and Theorem 4.12, we have $\theta_{1}+\theta_{2}=\frac{\pi}{2}$. This completes the proof of theorem.

Now, we need to following lemma: 
Lemma 5.3. Let $M$ be semi-slant or hemi-slant submanifold of a $S$ manifold. Then we have the following statements:

(1) For any $X \in \mathfrak{D}_{1}$ we have

$$
P_{2} T X=0
$$

(2) For any $X \in \mathfrak{D}_{2}$ we have

$$
P_{1} T X=0 .
$$

Proof. Suppose that $\left\{e_{1}, \ldots, e_{2 n}, e_{2 n+1}, \ldots, e_{2 n+2 m}, \xi_{1}, \ldots, \xi_{s}\right\}$ to be an orthonormal frame field on $T M$ where $\mathfrak{D}_{1}=\operatorname{Span}\left\{e_{1}, \ldots, e_{2 n}\right\}$,

$\mathfrak{D}_{2}=\operatorname{Span}\left\{e_{2 n+1}, \ldots, e_{2 n+2 m}\right\} \mathfrak{\mathbb { L }}=\operatorname{Span}\left\{\xi_{1}, \ldots, \xi_{s}\right\}$. Since $M$ is semi-invariant, we can choose this orthonormal frame field which satisfies

$$
\begin{aligned}
& T e_{1}=e_{2}, \ldots, T e_{2 n-1}=e_{2 n}, \\
& T e_{2 n+1}=\cos \theta_{2} e_{2 n+2}, T e_{2 n+2}=-\cos \theta_{2} e_{2 n+1}, \ldots, T e_{2 n+2 m}=-\cos \theta_{2} e_{2 n+2 m-1} .
\end{aligned}
$$

Therefore, we have $\widetilde{g}(f X, Y)=0$ when $X \in \mathfrak{D}_{1}$ and $Y \in \mathfrak{D}_{2}$ which shows the statements of (1) and (2) are also true for semi-slant submanifolds.

In a similar manner, it can be shown that the statements of $(i)$ and (ii) are true for hemi-slant submanifolds.

Corollary 5.4. Let $M$ be a $(2 n+2 m+s)$-dimensional semi-slant submanifold of an $S$ space form. Then, the following statements are true.

(1) For any unit vector field $X \in \mathfrak{D}_{1}$, it follows that

$$
\operatorname{Ric}(X) \leq \frac{1}{4} n^{2}\|H\|^{2}+\frac{c}{4}\{(2 n+2 m+2)\} .
$$

The equality case of (5.10) holds for all unit tangent vector $X \in \mathfrak{D}_{1}$ if and only if $M$ is $\mathfrak{D}_{1}$ totally geodesic point.

(2) For any unit vector field $X \in \mathfrak{D}_{2}$, it follows that

$$
\operatorname{Ric}(X) \leq \frac{1}{4} n^{2}\|H\|^{2}+\frac{c}{4}\left\{(2 n+2 m-1)+3 \cos ^{2} \theta_{2}\right\} .
$$

The equality case of (5.11) holds for all unit tangent vector $X \in \mathfrak{D}_{2}$ if and only if $M$ is $\mathfrak{D}_{2}$ totally geodesic point.

(3) The equality cases of both (5.10) and (5.11) do not satisfy.

Proof. Under the assumption, using $\theta_{1}=0$ and Lemma 5.3 in (5.6) and (5.7) we find (5.10) and (5.11). The equality cases of both (5.10) and (5.11) holds if and only if $M$ is $\mathfrak{D}_{1}$ and $\mathfrak{D}_{2}$ geodesic. Considering this fact and Corollary 4.14 we get a contraction. Thus the equality cases of both (5.10) and (5.11) do not satisfy.

Following the proof way of Corollary 5.4, we obtain the following corollary: 
Corollary 5.5. Let $M$ be a $(2 n+2 m+s)$-dimensional hemi-slant submanifold of an $S$ space form. Then, the following statements are true.

(1) For any unit vector field $X \in \mathfrak{D}_{1}$, it follows that

$$
\operatorname{Ric}(X) \leq \frac{1}{4} n^{2}\|H\|^{2}+\frac{c}{4}\left\{(2 n+2 m-1)+3 \cos ^{2} \theta_{1}\right\} .
$$

The equality case of (5.12) holds for all unit tangent vector $X \in \mathfrak{D}_{1}$ if and only if $M$ is $\mathfrak{D}_{1}$ totally geodesic.

(2) For any unit vector field $X \in \mathfrak{D}_{2}$, it follows that

$$
\operatorname{Ric}(X) \leq \frac{1}{4} n^{2}\|H\|^{2}+\frac{c}{4}\{(2 n+2 m-1)\} .
$$

The equality case of (5.13) holds for all unit tangent vector $X \in \mathfrak{D}_{2}$ if and only if $M$ is a $\mathfrak{D}_{2}$ totally geodesic.

(3) The equality cases of both (5.12) and (5.13) do not satisfy.

\section{References}

1. Chen BY, Geometry of slant submanifolds, Leuven: Katholieke Universiteit; 1990.

2. Atçeken M, Hui SK, Slant and pseudo-slant submanifolds in LCS-manifolds, Czech Math J. 2013; 61(1): $177-90$.

3. Carriazo A, Fernández LM, Hans-Uber MB, Some slant submanifolds of S-manifolds, Acta Math Hung. 2005; 107(4): 267-85.

4. Chen BY, Tazawa Y, Slant submanifolds of complex projective and complex hyperbolic spaces, Glasg Math J. 2000; 42(3): 439-54.

5. Gupta RS, Haider SMK, Sharfuddin A, Slant submanifolds in cosymplectic manifolds, Colloq Math. 2006; 105: 207-19.

6. Sahin B, Slant submanifolds of an almost product Riemannian manifold, J Korean Math Soc. 2006; 43(4): 717-32.

7. Carriazo A, Bi-slant immersions, Proc ICRAMS. 2000; 55: 88-97, 9th printing.

8. Carriazo A, New developments in slant submanifolds theory, Applicable Math Golden Age. 2002: 339-56.

9. Alegre P, Carriazo A (2019), Bi-slant submanifolds of para Hermitian manifolds, Multidisci Dig Pub Inst; 7(7): 618.

10. Gülbahar M, Kilıç E, Keleş S, A useful orthonormal basis on bi-slant submanifolds of almost Hermitian manifolds, Tamkang J Math. 2016; 47(2): 143-61.

11. Malek F, Balgeshir MBK, Semi-slant and bi-slant submanifolds of almost contact metric 3structure manifolds, Turk J Math. 2013; 37(6): 1030-39.

12. Özgür C, Siddiqu, AN, An integral formula for bi-slant submanifolds in complex space forms, Hacettepe J Math Stat. 2020; 49(6): 2028-36.

13. Perktaş SY, Blaga AM, Kılıç E, Almost bi-slant submanifolds of an almost contact metric manifold, J Geometry. 2021; 112(1): 1-23. 
14. Uddin S, Lone MS, Lone MA, Chen's $\delta$-invariants type inequalities for bi-slant submanifolds in generalized Sasakian space forms, J Geometry Phys. 2021; 161: 104040.

15. Chen BY, Relations between Ricci curvature and shape operator for submanifolds with arbitrary codimensions, Glasg Math J.. 1990; 41(1): 33-41.

16. Chen BY, Ricci curvature of real hypersurfaces in complex hyperbolic space, Arch Math. 2002; 38(1): 73-80.

17. Kılıç E, Tripathi MM, Gülbahar M, Chen-Ricci inequalities for submanifolds of Riemannian and Kaehlerian product manifolds, Ann Polonici Mathematici. 2016; 116: 37-56.

18. Mihai A, Özgür C, et al., Chen inequalities for submanifolds of real space forms with a semisymmetric metric connection, Taiwan J Math. 2010; 14(4): 1465-77.

19. Mustafa A, Uddin S, Al-Solamy FR, Chen-Ricci inequality for warped products in Kenmotsu space forms and its applications, Revista de la Real Academia de Ciencias Exactas, Físicas y Naturales. Serie A Matemáticas. 2010; 113(4): 3585-602.

20. Tripathi MM, Improved Chen-Ricci inequality for curvature-like tensors and its applications, Differ Geom Appl. 2011; 29(5): 685-98.

21. Yano K, Kon M, Structures on manifolds, World scientific. 1985; 3.

22. Hong S, Matsumoto K, Tripathi MM, et al., Certain basic inequalities for submanifolds of locally conformal Kaehler space forms, Sut J Math. 2005; 41(1): 75-94.

23. Blair DE, et al., Geometry of manifolds with structural group $U(n) \times O(s)$, J Differential Geometry. 1970; 4(2): 155-67.

24. Kobayashi M, Tsuchiya S, Invariant submanifolds of an f-manifold with complemented frames, Kodai Math Seminar Reports. 1992; 24(4): 430-50.

25. Cabrerizo JL, Fernandez LM, Fernandez M, The curvature of submanifolds of an $S$-space form, Acta Math Hung. 1993; 62(3-4): 373-83.

26. Cabrerizo JL, Carriazo A, Fernandez LM, Fernandez M, Slant submanifolds in Sasakian manifolds, Glasg Mat J. 2000; 42(1): 125-38.

\section{Corresponding author}

Aykut Akgün can be contacted at: muslumakgun@adiyaman.edu.tr

For instructions on how to order reprints of this article, please visit our website:

www.emeraldgrouppublishing.com/licensing/reprints.htm

Or contact us for further details: permissions@emeraldinsight.com 